\title{
Analysis on the Serious Adverse Effects Caused by Dietary Supplements
}

\author{
Xingjian Xie \\ School of science, Rensselaer Polytechnic Institute, Troy, New York, 12180 \\ ${ }^{*}$ Corresponding author. Email: xiex6@rpi.edu
}

\begin{abstract}
Many people in the US take dietary supplements regularly. Therefore, it is important to know if these products have any adverse effects. Additionally, due to the lack of strict regulation of supplements from the Food and Drug Administration (FDA), there is no evidence proving that some supplements have a positive effect on diseases. This paper illustrates some serious adverse effects after taking dietary supplements by employing statistical analysis and case study, drawing a conclusion that some supplements do cause adverse effects, and well-nourished people should consider seriously upon whether to take supplements or not since some supplements may only have adverse effects on them.
\end{abstract}

Keywords: Dietary supplements, Adverse effects, Cardiovascular diseases, Asthma, Type 2 diabetes, Liver injury

\section{INTRODUCTION}

The Food and Drug Administration (FDA) is a federal agency of the Department of Health and Human Services. It is responsible to regulate all food and drugs to ensure that they are safe for humans to take and drugs should be effective. According to the Dietary Supplement Health and Education Act (DSHEA) of 1994, a dietary supplement is defined as a product that is intended to supplement the diet containing at least one of the following ingredients: an amino acid, a mineral, a vitamin, an herb or other botanical. A dietary supplement should also be in the form of liquid, tablet, pill, or capsule and be labeled as a dietary supplement [1]. Also, FDA states that a disclaimer is allowed to be labeled on the products saying that "This statement has not been evaluated by the Food and Drug Administration. This product is not intended to diagnose, treat, cure, or prevent any disease [2]." Due to the difficulty of categorizing supplements as food or drug, they are not regulated as strictly as drugs and no clinical trials are being required.

It is true that supplements can provide people with vitamins and minerals, and the lack of vitamins and minerals over a period of time can cause diseases such as Scurvy, Rickets, and etc. However, people who have balanced diets are sufficient to intake enough vitamins and minerals to keep healthy. Moreover, due to the lack of strict regulations, there is no scientific evidence proving that supplements can prevent, treat, or cure any kind of disease [3]. Not only do some of the supplements have no obvious positive effect on disease, but they even cause harm to humans. This paper employed some cases and relevant data to analyze the adverse effects of supplements from different perspectives such as age, sex and product type, and listed several specific adverse effects caused by some dietary supplements. This paper aims to explore some serious adverse effects caused by dietary supplements and provide suggestions on the consumption and regulations of supplements in order to reduce the adverse effect cases caused by supplements. This paper also explains why more strict regulations on supplements should be published. This may help stimulate further research on the mechanisms of supplements, thus giving conductive suggestions in solving people's safety problems.

\section{METHODS AND MATERIALS}

The data from January 2014 to December 2017 was acquired from the CFSAN Adverse Event Reporting System (CAERS) of FDA [4], and R was used as an analytical tool. The author extracted patients' age, sex, product type, and outcomes for Table 1 and FDA product descriptions for Table 2. Results from previous studies on specific adverse effects caused by supplements were also employed to give possible suggestions on how to reduce these cases. 


\section{FACTORS THAT CAUSE SUPPLEMENT ADVERSE EFFECTS}

\subsection{Sex}

Among the total of 30766 supplement adverse effect cases from 2014 to 2017, 77.4\% were suspect supplements and $22.6 \%$ are concomitant supplements. Females took a larger portion of the total cases $(54.2 \%)$ than males $(16.3 \%)$. Cases reported by females were more than 3 times higher than those reported by males. Due to the existence of cosmetic supplements in the product types, it can be reasonably inferred that the difference in the consumption of supplements may depend on sex because cosmetic supplements are mostly bought by women and consumers who use other supplements are equally distributed among the two sexes.

\subsection{Age}

The median age of males was 55 and the average was 52.7. The 25 th percentile was 35 and the 75 th percentile was 71 . Females had a median age of 52 and an average age of 51.1. Their 25th percentile was 37 and 75th percentile was 65 . Male reporters tend to be older than female reporters in general. Among all genders, people who were older than 60 took up a large portion of the cases $(26.3 \%)$ and young children and teenagers took the least part of the cases (only 3.5\% in total). In general, older people were more likely to be reported for supplement adverse effects than the youth. Two possible hypotheses can be raised according to this difference. First, it is likely that old people care more about their health so they are more interested in supplements. The more supplements the old people take, the more adverse effect cases there will be. It is also possible that younger people are more immune to the poisonous components in supplements. Even if the same number of the young and the old take the same type of supplement, more adverse effect cases will be reported from old people since the poisonous molecules do more harm to them.

\subsection{Product type}

People's favorite mineral and vitamin supplements took the majority part of all adverse effect cases (47.2\%). Cosmetics took the second large proportion of cases (38.7\%) and alcoholic beverage was the third frequently reported supplement $(2.0 \%)$. Cases reported from other types of supplements were all very low. Therefore, it can be concluded that vitamin supplements are the most widely used supplement type and cosmetics are mostly popular among women. Since these products cause so many adverse cases, people should consider carefully before taking them.

\subsection{Outcomes}

Among all the possible serious outcomes, medical importance and intervention required outcomes were two major compositions, taking up $36.6 \%$ and $32.6 \%$ respectively. The least likely outcome was the congenital anomaly case, only taking up $0.1 \%$ of all cases. It is also worth noticing that the supplements had caused 3698 $(12.0 \%)$ death cases and $4.2 \%$ of life-threatening cases. Supplements are generally taken to improve people's health. It is unexpected to find that they can send people into hospitals and can even kill people.

Table 1. Information of Individuals and Outcomes of Supplement Adverse Effects, CAERS 2014-2017.

\begin{tabular}{lc}
\hline & $\begin{array}{c}\text { Overall } \\
\text { (N=30766) }\end{array}$ \\
\hline Age & \\
1 to 10 & $497(1.6 \%)$ \\
10 to 20 & $574(1.9 \%)$ \\
20 to 30 & $2238(7.3 \%)$ \\
30 to 40 & $3324(10.8 \%)$ \\
40 to 50 & $3153(10.2 \%)$ \\
50 to 60 & $3911(12.7 \%)$ \\
above 60 & $8106(26.3 \%)$ \\
Not Given & $8963(29.1 \%)$ \\
Sex & \\
Not Given & $9085(29.5 \%)$ \\
F & $16676(54.2 \%)$ \\
M & $5005(16.3 \%)$ \\
Product Type & \\
CONCOMITANT & $6943(22.6 \%)$ \\
SUSPECT & $23823(77.4 \%)$ \\
Outcomes & \\
Congenital Anomaly & $28(0.1 \%)$ \\
Death & $3698(12.0 \%)$ \\
Disability & $1195(3.9 \%)$ \\
Hospitalization & $3271(10.6 \%)$ \\
Life Threatening & $1289(4.2 \%)$ \\
Medically Important & $11262(36.6 \%)$ \\
Intervention Required & $10023(32.6 \%)$ \\
\hline &
\end{tabular}


Table 2. Adverse Effect Cases by Product Descriptions.

\begin{tabular}{|c|c|}
\hline & $\begin{array}{c}\text { Overall } \\
(\mathrm{N}=30766)\end{array}$ \\
\hline \multicolumn{2}{|l|}{ Description } \\
\hline & $623(2.0 \%)$ \\
\hline Alcoholic Beverage & $8(0.0 \%)$ \\
\hline Baby Food Products & $43(0.1 \%)$ \\
\hline Bakery Prod/Dough/Mix/lcing & $277(0.9 \%)$ \\
\hline Beverage Bases/Conc/Nectar & $18(0.1 \%)$ \\
\hline Candy W/O Choc/Special/Chew Gum & $63(0.2 \%)$ \\
\hline Cereal Prep/Breakfast Food & $230(0.7 \%)$ \\
\hline Cheese/Cheese Prod & $45(0.1 \%)$ \\
\hline Choc/Cocoa Prod & $77(0.3 \%)$ \\
\hline Coffee/Tea & $116(0.4 \%)$ \\
\hline Cosmetics & $11892(38.7 \%)$ \\
\hline Dietary Conventional Foods/Meal Replacements & $283(0.9 \%)$ \\
\hline Dressing/Condiment & $34(0.1 \%)$ \\
\hline Egg/Egg Prod & $131(0.4 \%)$ \\
\hline Filled Milk/lmit Milk Prod & $42(0.1 \%)$ \\
\hline Fishery/Seafood Prod & $162(0.5 \%)$ \\
\hline Food Additives (Human Use) & $173(0.6 \%)$ \\
\hline Food Sweeteners (Nutritive) & $28(0.1 \%)$ \\
\hline Formula Raw Material & $4(0.0 \%)$ \\
\hline Formula, Form Unspecified & $5(0.0 \%)$ \\
\hline $\begin{array}{l}\text { Fruit/Fruit Prod } \\
\text { Gelatin/Rennet/Pudding Mix/Pie Filling }\end{array}$ & $\begin{array}{r}304(1.0 \%) \\
7(0.0 \%)\end{array}$ \\
\hline Ice Cream Prod & $93(0.3 \%)$ \\
\hline Liquid Concentrate Formula & $3(0.0 \%)$ \\
\hline Macaroni/Noodle Prod & $28(0.1 \%)$ \\
\hline Meat, Meat Products and Poultry & $15(0.0 \%)$ \\
\hline Medical Foods, N.E.C. & $76(0.2 \%)$ \\
\hline Milk/Butter/Dried Milk Prod & $122(0.4 \%)$ \\
\hline Miscellaneous Food Related Items & $1(0.0 \%)$ \\
\hline Mult Food Dinner/Grav/Sauce/Special & $138(0.4 \%)$ \\
\hline Nuts/Edible Seed & $111(0.4 \%)$ \\
\hline Powder Formula & $37(0.1 \%)$ \\
\hline Prep Salad Prod & $75(0.2 \%)$ \\
\hline Ready to Serve Formula & $14(0.0 \%)$ \\
\hline Snack Food Item & $167(0.5 \%)$ \\
\hline Soft Drink/Water & $252(0.8 \%)$ \\
\hline Soup & $27(0.1 \%)$ \\
\hline Spices, Flavors And Salts & $45(0.1 \%)$ \\
\hline Vegetable Oils & $15(0.0 \%)$ \\
\hline Vegetable Protein Prod & $14(0.0 \%)$ \\
\hline Vegetables/Vegetable Products & $382(1.2 \%)$ \\
\hline Vit/Min/Prot/Unconv Diet(Human/Animal) & $14528(47.2 \%)$ \\
\hline Whole Grain/Milled Grain Prod/Starch & $56(0.2 \%)$ \\
\hline Food Service/Conveyance & $2(0.0 \%)$ \\
\hline
\end{tabular}

\section{SPECIFIC ADVERSE EFFECTS OF SUPPLEMENTS}

\subsection{Liver injury}

In the experiment conducted by Victor J. Navarro and his partners on hepatotoxicity caused by herbals and dietary supplements and conventional medications in 2014 [5], they collected data from patients with at least two years of experiencing drug-induced liver injury within the past six months. Among the total of 845 eligible cases, 709 liver injury cases were due to medication and 136 cases were due to herbal and dietary supplements, which took up about $16 \%$ of the total cases and this number was gradually increasing from 2004 to 2012. the increasing number of people taking all kinds of supplements is one of the major causes of this increment. Also, most of the 136 patients took more than one type of supplement product and they took 217 products in total. This is also an implication that supplements can cause liver injury.

\subsection{Cardiovascular diseases}

Fish oil is often derived from oily fish and contains plenty of long chain omega-3 fatty acids eicosapentaenoic acid (EPA) and docosahexaenoic acid (DHA). They have been widely used as supplements to prevent cardiovascular diseases (CVD). However, fish oil was shown to cause CVD mortality by the experiment conducted by Zhi-Hao Li and his coworkers in 2020 [6].

There was a total number of 427,678 individuals with no CVD or cancer at baseline from age 40 to 69 participating in the experiment. Researchers used an electronic questionnaire to record participants' habitual use of fish oil supplements and $31.2 \%$ of them were reported to habitually use fish oil supplements. During the follow-up period with a median time of 9 years, there were 12,928 death cases in total. They found a strong association between fish oil supplement use and CVD mortality and myocardial infarction mortality.

\subsection{Type 2 diabetes}

Fish oil may also cause type 2 diabetes (T2D). In the research conducted by Guo-Chong Chen in 2021, 392,287 middle-aged and older individuals who were free of diabetes, cancers, and major cardiovascular diseases participated in this research [7]. Their habitual dietary intake information was collected for further analysis.

As a result, 7262 cases of T2D were identified during a median of 10.1 years follow-up. After doing some statistical analysis on the data they got, they found out that the consumption of oily fish was significantly inversely associated with T2D. Also, the regular intake of fish oil supplements was related to the risk of T2D.

\subsection{Asthma}

Maternal folic acid supplements are widely used prior to and during the first trimester of pregnancy in order to prevent infant neural tube defects. According to the experiment conducted by Whitrow in 2009, however, this kind of supplement can lead to asthma in children [8]. They conducted a cohort study $(\mathrm{n}=557)$ on pregnant women in Adelaide, South Australia from 1998 to 2005. 
Maternal dietary information and their children's asthma information were collected.

They found out that $11.6 \%$ of children in 3.5 years and $11.8 \%$ of children in 5.5 years were reported to have asthma. Folic supplements taken in late pregnancy were associated with the risk of childhood asthma with a relative risk of 1.26 .

\section{SUGGESTIONS ON THE REGULATION OF SUPPLEMENTS}

\subsection{Consumers}

Supplements are widely used around the world. Many consumers take them because they want to become healthier or they think it is the fashion to take supplements. They hardly realize that supplements are not totally harmless. Indeed, some of them are quite poisonous and can even cause death. Consumers should take serious account before using any kind of supplements. Supplements are not as strictly regulated as medicines by FDA. Most supplements are not designed for treating and preventing any kind of disease so they cannot take place of medicine. Supplements are effective to people with unbalanced diets but can also cause diseases. Both benefits and drawbacks should be considered before making the decision to take supplements.

\subsection{Manufacturers}

Manufacturers of supplements are responsible for the safety and effectiveness of their products. They are required to do tests on their products in order to ensure that the products are safe enough for consumers to use. This process, however, is not as strictly regulated as clinical trials required for medicines since supplements are in the category between food and medicine. Therefore, it is possible that some supplements products can pass the clinical trials required by FDA but still cause serious adverse effects. It is also very common that some supplements show positive effects in a small group test but their effects are not significant when used by a larger number of people. This can be one reason for the inconsistency between supplement effects and their advertisements. In order to avoid these problems, manufacturers should do more clinical trials on supplements in order to at least make sure their products do no harm to humans and products' effects are consistent with their advertisements. Exaggerations in advertisements should be limited. Warnings about the low risk of serious adverse effects labeled on the bottle of the products may be effective in reducing the cases of the adverse effects. These warnings can also encourage consumers to do more research before determining to use supplements.

\subsection{Government}

According to Dietary Supplement Labeling Guide from FDA in 2018 [9], a health claim is required to be labeled on the container of the product to show the relationship between substances in the product and a health-related condition. This type of claim is authorized by FDA, so the substances mentioned in these claims are relatively safe. However, a qualified health claim can be used without the authorization of the FDA. Only an agency-approved disclaimer is required. This can lead to a large portion of adverse effects caused by poisonous molecules in supplement products. The National Institutes of Health (NIH) offers many funding opportunities for the supplements research. According to the website of NIH [10], the maximum funding for supplement research is 10,000 dollars. In order to reduce the number of supplement adverse effect cases, the government should publish a more strict policy on the regulation of supplements, so that fewer supplements will contain harmful substances. Besides, the government can give more subsidies to both individuals and manufacturer companies to encourage them to do more researches and clinical trials supplements. It is also a good option to inform the general public that supplements cannot take over medicine and have potential adverse effects.

\section{CONCLUSION}

Dietary supplements are largely used by the general public. More than half of the people in the US take dietary supplements. People choose to take supplements in the wish of improving their health conditions. However, due to the lack of strict regulation on supplements by the FDA, many of them are not capable of treating or preventing diseases. Some supplements have serious adverse effects including T2D, asthma, liver injury, cardiovascular disease and etc., and can even cause death. People should consider seriously before deciding to take any kind of supplements. The government and manufacturing companies should take actions to improve the safety standards of supplement products. This paper only gives the possible adverse effects caused by supplements but the detailed mechanisms are still not fully explained. Future research can focus on the mechanisms of how poisonous molecules in supplements can cause diseases. If these mechanisms can be fully understood, it is more likely for the manufacturers to produce safer supplements.

\section{REFERENCES}

[1] Mueller, C. (1999). The regulatory status of medical foods and dietary supplements in the United States. Nutrition, 15(3), 249-251. doi:10.1016/s08999007(98)00186-5.

[2] CFR - Code of Federal Regulations Title 21. 
[3] Gurley BJ, Yates CR, Markowitz JS. "... Not Intended to Diagnose, Treat, Cure or Prevent Any Disease." 25 Years of Botanical Dietary Supplement Research and the Lessons Learned. Clin Pharmacol Ther. 2018 Sep;104(3):470-483. doi: 10.1002/cpt.1131. Epub 2018 Jul 13. PMID: 29882958.

[4] CFSAN Adverse Event Reporting System (CAERS) https://www.fda.gov/food/complianceenforcement-food/cfsan-adverse-event-reportingsystem-caers\#files.

[5] Navarro VJ, Barnhart H, Bonkovsky HL, et al. Liver injury from herbals and dietary supplements in the U.S. Drug-Induced Liver Injury Network. Hepatology. 2014;60(4):1399-1408. doi:10.1002/hep. 27317.

[6] Li ZH, Zhong WF, Liu S, et al. Associations of habitual fish oil supplementation with cardiovascular outcomes and all cause mortality: evidence from a large population based cohort study. BMJ. 2020;368:m456. Published 2020 Mar 4. doi:10.1136/bmj.m456.

[7] Chen GC, Arthur R, Qin LQ, Chen LH, Mei Z, Zheng Y, Li Y, Wang T, Rohan TE, Qi Q. Association of Oily and Nonoily Fish Consumption and Fish Oil Supplements With Incident Type 2 Diabetes: A Large Population-Based Prospective Study. Diabetes Care. 2021 Mar;44(3):672-680. doi: 10.2337/dc20-2328. Epub 2021 Jan 11. PMID: 33431419; PMCID: PMC7896269.

[8] Whitrow, M. J., Moore, V. M., Rumbold, A. R., \& Davies, M. J. (2009). Effect of Supplemental Folic Acid in Pregnancy on Childhood Asthma: A Prospective Birth Cohort Study. American Journal of Epidemiology, 170(12), 1486-1493. doi:10.1093/aje/kwp315.

[9] Dietary Supplement Labeling Guide https://www.fda.gov/food/dietary-supplementsguidance-documents-regulatoryinformation/dietary-supplement-labeling-guide.

[10] Research Supplements: Investigators developing independent research careers https://www.niaid.nih.gov/grantscontracts/research-supplements. 